\title{
Community Newsletter: Spectrum survey on 2021 conferences, Bayesian precision, paper review feedback
}

\section{BY CHELSEY B. COOMBS}

19 SEPTEMBER 2021

Hello, and welcome to this week's Community Newsletter! I'm your host, Chelsey B. Coombs, Spectrum's engagement editor.

Before we get started, I want to let you know about a new Spectrum survey. We are interested in learning how autism researchers are approaching conferences through the end of the year. Are you ready to get back to the crowds at this season's conferences? Or will you be watching the action from your lab (or couch)? Let us know, and look out for a Spectrum article on how the field is thinking about in-person meetings going forward.

Online this week, Daniel Yon, lecturer in psychology at Goldsmiths, University of London in the United Kingdom, and Chris Frith, emeritus professor of neuropsychology at University College London, wrote about how precision is important to ideas about the 'Bayesian brain.'

When Bayesian brain theories talk about 'precision', what precisely do they mean? In @CurrentBiology @cdfrith \& I explain how precision has shaped thinking about perception, learning, metacognition \& social interaction. But some key puzzles remain unsolved https://t.co/IYV6SvSilq

— Daniel Yon (@danieljamesyon) September 13, 2021

The Bayesian brain theory holds that people and animals weigh prior knowledge and incoming 


\section{Spectrum | Autism Research News}

https://www.spectrumnews.org

information in terms of reliability - and they take action based on what they deem most reliable, or precise. It's all about estimating uncertainty, the authors write, and it has direct relevance to autism.

"Characteristics of autism, such as a preference for stable and repetitive environments, can be cast as a consequence of overly strong beliefs about the precision of incoming evidence, such that every fluctuation in our sensory systems seems to signal the need to change our models of the environment (and the world thus seems unstable)," the authors write.

Autism researcher Uta Frith, emeritus professor of cognitive development at University College London, who is married to Chris Frith, tweeted about the study.

A primer explaining why 'precision' has become such an important term when theorising how the brain works.

Access the final version of the paper for free (until 2nd Nov) via this share link:

https://t.co/yC8FizH2uT... https://t.co/nHQSPjOJS3

— Uta Frith (@utafrith) September 14, 2021

Micah Allen, associate professor of clinical medicine at Aarhus University in Denmark, praised the paper.

An extremely useful paper - well done!

— Micah Allen (@micahgallen) September 13, 2021

Sven De Maeyer, professor of education and education sciences at the University of Antwerp in Belgium, tweeted about how the paper relates to Bayesian statistical modelling.

Nice primer on a topic I was less familiar with. Also interesting read to make analogies when talking about bayesian statistical modelling. https://t.co/PYIYqfMqunh 


\section{Spectrum | Autism Research News}

https://www.spectrumnews.org

— Sven De Maeyer (@svawa) September 13, 2021

Our next thread this week comes from David Mandell, professor of psychiatry at the University of Pennsylvania and Autism editor-in-chief. He tweeted that journal reviewers should be kind as they "shape the next generation" of academics.

I'm a journal editor. Got a review back for a paper that was fair but harsh. I softened the language before sending it back. I rejected the paper, but the author, who is a student, thanked me for the helpful comments. Reviewers, be kind. Your words shape the next generation.

_David Mandell (@DSMandell) September 15, 2021

Many researchers chimed in with their own experiences with reviewers.

Naomi Ekas, associate professor of psychology at the Texas Christian University in Fort Worth, tweeted about the harsh review of a paper that eventually became her most cited.

I once got a R\&r and the editor said "ignore reviewer 2 , but just quickly address reviewer 3." Reviewer 2 had a sentence that just said "this isn't good enough. Reject." Soooo helpful ???? oddly enough, it's my most cited paper now

—NaomiEkas (@EkasNaomi) September 16, 2021

Alycia Halladay, chief science officer at the Autism Science Foundation, tweeted, "You are my inspiration for feedback to ASF applications."

Thank you! You are my inspiration for feedback to ASF applications. There is something good about all the applications we receive, we just can't fund them all, and want fellows to keep trying and not get discouraged. It's a jungle out there. 


\section{Spectrum | Autism Research News}

https://www.spectrumnews.org

—Alycia Halladay (@AHalladayASF) September 15, 2021

Jessica Dark, a graduate student in organizational psychology at Birbeck, University of London, wrote about her own positive review experience with an autism journal.

It seems I have been so lucky so far. I sent my masters research for review at a well known autism journal. They rejected it and explained why, but they also gave encouragement regarding my writing and asked me to resubmit future work. How reviews should be!

—_Jessica Dark PhD (@phd_dark) September 16, 2021

Don't forget to register for our 28 September webinar, featuring Jeremy Veenstra-VanderWeele, professor of psychiatry at Columbia University, who will speak about goals for developing new drugs for autism - and the barriers researchers may encounter.

That's it for this week's Community Newsletter! If you have any suggestions for interesting social posts you saw in the autism research sphere, feel free to send an email to me at chelsey@spectrumnews.org. See you next week!

Cite this article: https://doi.org/10.53053/NCYL2116 